\title{
Psoas density - an optimal sarcopaenic indicator associated with postoperative complications after colorectal resection for cancer?
}

\author{
Anna Pekařová1,2, Matej Pekař ${ }^{1,3}$, Marek Soltes ${ }^{4}$, Lucia Havrlentová ${ }^{3}$, Tereza Chovancová ${ }^{3,5}$ \\ ${ }^{1}$ Department of Physiology, Faculty of Medicine, Masaryk University, Brno, Czech Republic \\ ${ }^{2}$ Lung Disease Department, Faculty Hospital, Ostrava, Czech Republic \\ ${ }^{3}$ Department of Surgery, Vitkovice Hospital, Ostrava, Czech Republic \\ ${ }^{4} 1^{\text {st }}$ Department of Surgery, University of Pavol Jozef Safarik, Kosice, Slovak Republic \\ ${ }^{5}$ Department of Surgery, Faculty of Medicine, University of Ostrava, Ostrava, Czech Republic
}

Videosurgery Miniinv 2021; 16 (1): 91-97

DOI: https://doi.org/10.5114/wiitm.2020.100880

\begin{abstract}
Introduction: Sarcopaenia seems to be predictive factor for postoperative morbidity and mortality after colorectal resection for cancer. Nevertheless, an ideal sarcopaenic indicator is still to be identified.

Aim: To evaluate computed tomography (CT) measured total abdominal muscle area (TAMA), total psoas muscle area (TPA), and psoas density (PD) - previously described sarcopaenia indicators - as possible risk factors for postoperative complications in patients after curative colon and rectal resections for colorectal cancer.

Material and methods: Consecutive patients after elective curative colon or rectal resection for cancer at a single institution were divided into cohorts with uncomplicated postoperative course or complications Clavien-Dindo grade I-II (Cl-Di O-II) and complications Clavien-Dindo grade III-V (Cl-Di III-V). Cohorts were statistically tested for significant differences in TAMA, TPA, and PD calculated from preoperative staging CT scans at the level of the third lumbar vertebra.

Results: Data of 112 patients were analysed from a prospectively run database; 65 underwent colon and 47 rectal resections. PD was significantly higher in the Cl-Di O-II cohort compared to the Cl-Di III-V for both colon (42.67 \pm 6.52 vs. $40.11 \pm 7.57 \mathrm{HU}, p=0.002)$ and rectal resections ( $44.08 \pm 5.86$ vs. $43.03 \pm 5.70 \mathrm{HU}, p=0.016)$. TAMA and TPA failed to show significant differences.

Conclusions: Psoas density is significantly decreased in patients with Clavien-Dindo grade III-V complications after curative resection for colon and rectal cancer. Due to the simplicity and affordability of its assessment from preoperative staging CT scan, it might be considered an optimal sarcopaenic indicator to be utilised in everyday practice.
\end{abstract}

Key words: complications, colorectal cancer, computed tomography, sarcopaenia, psoas density.

\section{Introduction}

Colorectal cancer represents serious disease with steadily growing incidence worldwide, leading to significant socio-economic implications both for the patients and society. Despite advances in oncological treatment and technical progress in surgical techniques, it remains the second most deadly cancer [1]. Although surgical treatment is considered highly standardised and effective, numerous postoperative complications resulting in serious morbidity and even mortality are consistently reported [2]. Therefore, every effort should be made to improve this situation.

\footnotetext{
Address for correspondence

Marek Soltes Assoc. Prof., MD, PhD, $1^{\text {st }}$ Department of Surgery, University of Pavol Jozef Safarik, Kosice, Slovak Republic,

e-mail: soltes.marek@yahoo.com
} 
To allow more appropriate comparison of data regarding surgical complications, the Clavien-Dindo classification was introduced in 2004 as a global compelling tool for quality assessment in surgery. The proposed morbidity scale, based on the therapeutic consequences of complications, is simple, objective, and reproducible [3]. As a result, the previously utilised subjective description of minor (Clavien-Dindo grade I-II) and major complications (Clavien-Dindo grade III or more) should no longer be acceptable within surgical research and publishing.

Based on the current guidelines of the European Working Group on Sarcopaenia in Older People (EWGSOP2), sarcopaenia is defined as muscle disease characterised by low muscle strength associated with low muscle quantity or quality. Possible association with low physical performance is an indicator of severity [4]. While loss of muscle mass beneath the normal values (quantity) is relatively straightforward, impaired quality appears to be defined more vaguely. Nevertheless, sarcopaenic obesity caused by fat infiltration of muscle tissue that replaces its volume may be considered a good example $[5,6]$.

Both sarcopaenia and sarcopaenic obesity increase health risks and are associated with cardiac and respiratory diseases, impaired cognitive functions, reduced physical performance, and increased direct hospital costs [4]. Sarcopaenia also seems to be a poor prognostic factor for survival of cancer patients, independent of body mass index [7]. Also of note, there is some evidence that sarcopaenia could be a predictive factor for postoperative surgical complications in colorectal surgery [8-10]. Nevertheless, preoperative assessment of sarcopaenia is not utilised on a regular standardised basis, and its true value for prediction of postoperative outcome remains controversial. This situation is clearly reflected in the EWGSOP2 guidelines, which declare that the search for muscle quality indicators that best predict outcomes and identification of measuring tools that are affordable represent gaps in sarcopaenia research [4].

\section{Aim}

The aim of the study was to evaluate computed tomography (CT) measured total abdominal muscle area (TAMA), total psoas muscle area (TPA), and psoas density (PD) - previously described sarcopenia indicators - as possible risk factors for postoperative complications in patients after curative colon and rectal resections for colorectal cancer.

\section{Material and methods}

After the approval of the Multicentre Ethics Committee (ethical approval number: EK/279/2019), in accordance with the Declaration of Helsinki, all patients who underwent elective curative colon or rectal resection for colorectal cancer at our institution between 1.1.2017 and 31.12.2018 were identified in the prospectively run database. Rectal cancer was defined as a tumour within $15 \mathrm{~cm}$ of the anal verge. To assure homogenous quality of the CT scans utilised for subsequent analysis, patients with staging CT performed outside our institution were excluded from the study protocol.

Demographic and clinical data of the study population including age, sex, body mass index (BMI), American Society of Anaesthesiologists (ASA) score, neoadjuvant chemoradiotherapy, type of surgical approach (open/laparoscopic), postoperative complications according to Clavien-Dindo classification, 30-day mortality, and 1-year survival were collected/ calculated by a single independent clinical researcher blinded to the outcomes of CT muscle mass and density measurements.

Assessment of the standardised staging $\mathrm{CT}$ scans was executed by a single operator, skilled in radiological anatomy and body composition analysis, blinded to the patient outcomes to avoid bias and possible inter-observer variation. TAMA, TPA, and average PD were assessed at the cross sections in the third lumbar vertebra (L3) level where both transverse processes were visible. For TAMA and TPA, freehand drawing around the muscle margins was performed (TAMA - psoas, quadratus lumborum, erector spinae, external and internal obliques, transversus and rectus abdominis, TPA - psoas), while for PD freehand drawing $3 \mathrm{~mm}$ centrally to the psoas margin was done to avoid density variations at the borderline with surrounding tissues. Non-contrast CT images were used for the analysis to exclude contrast as a possible confounder. Calculations were then executed automatically by IntelliSpace Portal 9.0 software (Philips) - muscle attenuation in Hounsfield units (HU) for PD and $\mathrm{cm}^{2}$ for TAMA and TPA. Final values of TAMA and TPA in $\mathrm{cm}^{2} / \mathrm{m}^{2}$ were subsequently calculated as the proportion to the square of body height. 
Due to the fact that colon and rectal cancer represent two relatively different entities with regards to therapeutic strategies and outcomes, the study population was divided according to tumour localisation into two arms - "colon" and "rectum". Based on the recorded postoperative course, each arm was subsequently subdivided into two cohorts: Cl-Di 0-II (patients with no complications or complications Clavien-Dindo grade I-II) and Cl-Di III-V (complications Clavien-Dindo grade III-V).

\section{Statistical analysis}

Finally, statistical analysis was carried out with the aid of statistical software (Statistica 12, StatSoft, $\mathrm{CZ}$ ) to detect possible significant differences in muscle mass and density measurements (TAMA, TPA, and PD) between cohorts in both arms. A non-parametric Mann-Whitney $U$-test was utilised to compare unpaired continuous data. For the remaining variables Mann-Whitney $U$-test (continuous data) or Fischer's exact test (categorical data) was used for statistical analysis where applicable. The level of statistical significance was set to $p<0.05$.

\section{Results}

Overall, 118 patients with histologically confirmed colorectal adenocarcinoma and elective cura- tive resection (RO) were identified over the two-year period in the prospectively run database. Six cases were excluded due to preoperative CT scan performed at a different institution, which resulted in 112 patients being eligible for the study. The male/ female ratio was approximately $2: 1$, and the average BMI was $28.29 \pm 5.62 \mathrm{~kg} / \mathrm{m}^{2}$ (overweight range), $36.2 \%$ of rectal cancer cases underwent neoadjuvant chemoradiotherapy. $90.2 \%$ of patients were classified as ASA score $2-3$, and $83 \%$ of surgeries were performed by laparoscopic approach. Anastomotic leakage occurred in 6.3\%, 30-day mortality was $5.36 \%$, and 1 -year survival reached $92.9 \%$. The colon and rectum arms included 65 and 47 patients, respectively. Basic descriptive data of the study population are summarized in Table I - continuous variables are presented as mean values with standard deviation, categorical ones as total numbers and proportion.

No significant difference was detected between Cl-Di 0-II and Cl-Di III-V cohorts in age, gender, BMI, ASA score, and neoadjuvant chemoradiotherapy in both colon and rectum arms. In the colon arm the Cl-Di 0-Il cohort significantly more often received laparoscopic procedure ( $87.8 \%$ vs. $37.5 \%, p=0.0002$ ) while the $\mathrm{Cl}-\mathrm{Di}$ III-V cohort more often received open surgery $(62.5 \%$ vs. $12.2 \%, p=0.0002)$. This difference, however, does not compromise subsequent

Table I. Basic descriptive data

\begin{tabular}{|c|c|c|c|}
\hline Basic characteristics & Total & Colon & Rectum \\
\hline Number of patients & 112 & 65 & 47 \\
\hline Gender ratio (male/female) & $74 / 38(66 / 34 \%)$ & $40 / 25(62 / 38 \%)$ & $34 / 13(72 / 28 \%)$ \\
\hline Age [years] & $68.3 \pm 10.4$ & $68.5 \pm 10.8$ & $68.0 \pm 9.8$ \\
\hline Body mass index $\left[\mathrm{kg} / \mathrm{m}^{2}\right]$ & $28.29 \pm 5.62$ & $28.51 \pm 5.86$ & $27.98 \pm 5.25$ \\
\hline \multicolumn{4}{|l|}{ Comorbidity, $n(\%)$ : } \\
\hline ASA 1 & $8(7.1)$ & $6(9.2)$ & $2(4.3)$ \\
\hline ASA 2 & $54(48.2)$ & $28(43.1)$ & $26(55.3)$ \\
\hline ASA 3 & $47(42)$ & $28(43.1)$ & $19(40.4)$ \\
\hline ASA 4 & $3(2.7)$ & $3(4.6)$ & 0 \\
\hline \multicolumn{4}{|l|}{ Therapeutic approach, $n$ (\%): } \\
\hline Laparoscopic resection & $93(83)$ & $49(75)$ & $44(93.6)$ \\
\hline Open resection & $19(17)$ & $16(25)$ & $3(6.4)$ \\
\hline Neoadjuvant chemoradiotherapy & - & - & $17(36.2)$ \\
\hline
\end{tabular}


analysis because sarcopaenia is not related to the selection of surgical approach. A detailed overview is provided in Tables II and III.

PD was significantly higher in the Cl-Di 0-II cohort compared to Cl-Di III-V for both colon (42.67 \pm 6.52 vs. $40.11 \pm 7.57 \mathrm{HU}, p=0.002)$ and rectal resections ( $44.08 \pm 5.86$ vs. $43.03 \pm 5.70 \mathrm{HU}, p=0.016)$.
TAMA and TPA failed to show significant differences - Table IV, Figures 1 and 2.

Patients with anastomotic leakage after colon resection had significantly lower PD compared to those without leak ( $31.40 \pm 7.50$ vs. $42.73 \pm 6.37, p=$ 0.034 ), while no such difference was observed in patients after rectal resection. TAMA and TPA failed to

Table II. Colon arm cohorts

\begin{tabular}{|c|c|c|c|}
\hline Basic characteristics & Cl-Di 0-II & Cl-Di III-V & $P$-value \\
\hline Number of patients & 49 & 16 & - \\
\hline Gender ratio (male/female) & $30 / 19(61 / 39 \%)$ & $10 / 6(62.5 / 37.5 \%)$ & NS \\
\hline Age [years] & $68.0 \pm 10.5$ & $69.8 \pm 11.4$ & NS \\
\hline Body mass index $\left[\mathrm{kg} / \mathrm{m}^{2}\right]$ & $28.22 \pm 5.63$ & $29.39 \pm 6.41$ & NS \\
\hline \multicolumn{4}{|l|}{ Comorbidity, $n(\%)$ : } \\
\hline ASA 1 & $6(12)$ & 0 & \multirow[t]{4}{*}{ NS } \\
\hline ASA 2 & $23(47)$ & $5(31)$ & \\
\hline ASA 3 & $19(39)$ & $9(56)$ & \\
\hline ASA 4 & $1(2)$ & $2(13)$ & \\
\hline \multicolumn{4}{|l|}{ Therapeutic approach, $n(\%)$ : } \\
\hline Laparoscopic resection & $43(87.8)$ & $6(37.5)$ & \multirow[t]{2}{*}{0.0002} \\
\hline Open resection & $6(12.2)$ & $10(62.5)$ & \\
\hline Neoadjuvant chemoradiotherapy & - & NS & - \\
\hline
\end{tabular}

Table III. Rectum arm cohorts

\begin{tabular}{|lccc|}
\hline Basic characteristics & Cl-Di 0-II & Cl-Di III-V & $P$-value \\
\hline Number of patients & 40 & 7 & - \\
\hline Gender ratio (male/female) & $29 / 11(72.5 / 27.5 \%)$ & $5 / 2(71.4 / 28.6 \%)$ & NS \\
\hline Age [years] & $67.7 \pm 9.8$ & $69.9 \pm 9.5$ & NS \\
\hline Body mass index $\left[\mathrm{kg} / \mathrm{m}^{2}\right]$ & $27.96 \pm 5.41$ & $28.11 \pm 4.28$ & NS \\
\hline $\begin{array}{l}\text { Comorbidity, } n(\%): \\
\text { ASA 1 }\end{array}$ & $23(57.5)$ & $3(42.9)$ & \\
\hline ASA 2 & $15(37.5)$ & $4(57.1)$ & 0 \\
\hline ASA 3 & 0 & & NS \\
\hline ASA 4 & $38(95)$ & $1(14.3)$ & NS \\
\hline Therapeutic approach, $n$ (\%): & $2(5)$ & $3(42.8)$ & \\
\hline Laparoscopic resection & $14(35)$ & & \\
\hline Open resection & & & \\
\hline Neoadjuvant chemoradiotherapy &
\end{tabular}


show any significant differences in either arm. Nevertheless, analysis of anastomotic leakage was not a primary endpoint of the study because the number of patients was far too small to provide any reliable conclusions.

\section{Discussion}

There seems to be growing evidence that sarcopaenia is associated with postoperative complications and decreased survival in cancer patients [7-12]. Although this is not necessarily causation, because low muscle strength and low muscle quantity/quality may be related to age, gender, level of physical activity, heritability, and other possible confounders [13], it appears that some sarcopaenic indicators might be effective predictors of postoperative morbidity and mortality after colorectal resections for cancer [8-10]. At the same time, optimal parameters best predicting outcome and accurate affordable measuring tools are still to be defined [4].

$\mathrm{CT}$, magnetic resonance imaging (MRI), dual-energy X-ray absorptiometry (DXA), and bioelectrical impedance analysis (BIA) have been reported as validated tools for sarcopaenia assessment, all with specific pros and cons. Taking into account the disputable accuracy of BIA and inability of DXA to assess intra-muscular fat (muscle quality) [14], CT and MRI currently represent the gold standard to describe skeletal muscle quantity and quality [4, 15].

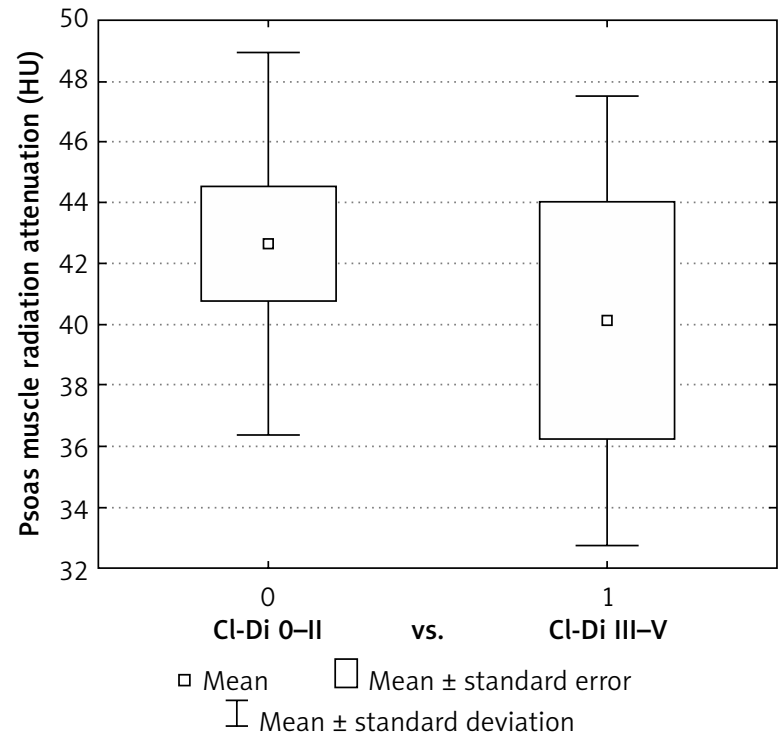

Figure 1. Psoas muscle density and complications (colon cancer)
Table IV. TAMA, TPA, and PD comparison between cohorts

\begin{tabular}{|c|c|c|c|}
\hline Variable & $\begin{array}{c}\text { TAMA } \\
{\left[\mathrm{cm}^{2} / \mathrm{m}^{2}\right]}\end{array}$ & $\begin{array}{c}\text { TPA } \\
{\left[\mathrm{cm}^{2} / \mathrm{m}^{2}\right]}\end{array}$ & PD (HU) \\
\hline \multicolumn{4}{|l|}{ Colon cancer: } \\
\hline Cl-Di 0-II, $n=49$ & $48.62 \pm 9.82$ & $5.63 \pm 1.83$ & $42.67 \pm 6.52$ \\
\hline Cl-Di III-V, $n=16$ & $48.64 \pm 9.38$ & $5.37 \pm 1.82$ & $40.11 \pm 7.57$ \\
\hline$P$-value & 0.2 & 0.99 & 0.002 \\
\hline \multicolumn{4}{|l|}{ Rectal cancer: } \\
\hline Cl-Di 0-II, $n=40$ & $50.50 \pm 11.82$ & $5.96 \pm 1.64$ & $44.08 \pm 5.86$ \\
\hline Cl-Di III-V, $n=7$ & $51.55 \pm 9.68$ & $6.51 \pm 1.78$ & $43.03 \pm 5.70$ \\
\hline P-value & 0.67 & 0.70 & 0.016 \\
\hline
\end{tabular}

In cancer patients, preoperative staging CT/MRI is routinely performed, so data for analysis are readily available with no need for any additional investigations or extra costs. Lumbar muscle cross-sectional area analysis, performed at the level of L3, was proposed as an innovative, simple, and practical tool to be adopted in clinical practice [4]. Therefore, this technique was selected for the presented study.

As for the sarcopaenic indicators assessed radiologically at the L3 level, the vast majority of studies report TAMA or TPA that were shown to correlate with the total body skeletal muscle volume [16]. Both parameters provide information about the muscle

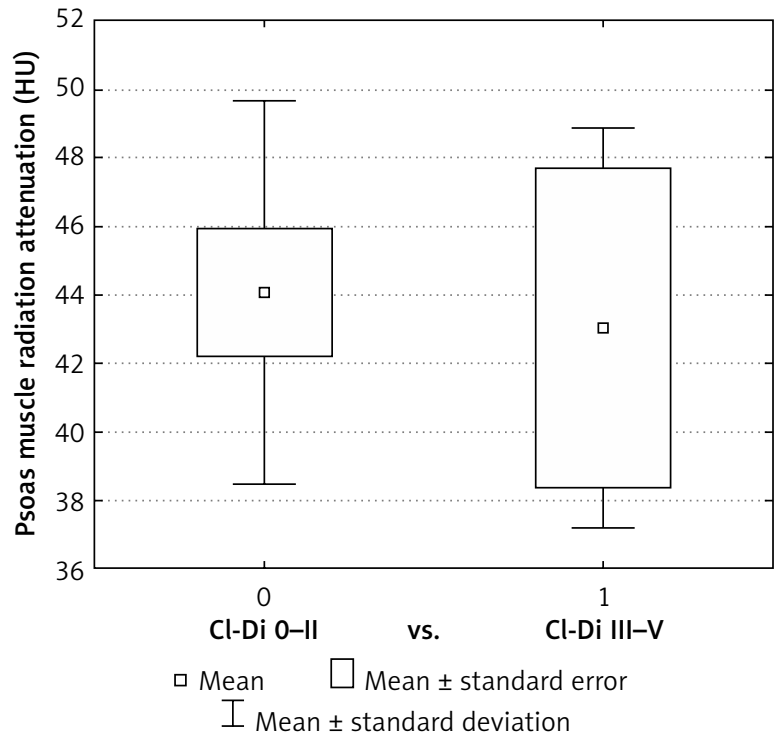

Figure 2. Psoas muscle density and complications (rectal cancer) 
quantity, thus reflecting the original definition of sarcopaenia. Nevertheless, novel measures are needed to reflect also muscle quality [17]. PD, defined as psoas muscle radiation attenuation measured from the CT scan in HU, indicates the ectopic lipid deposits in muscles that are not visually detectable as fat in the CT image, thus providing information about muscle quality [5]. Based on the above, the decision was taken to observe traditional TAMA and TPA, as well as more novel PD, as the indicators of sarcopaenia in our study. Decreased TAMA, TPA, and PD values were expected in the Cl-Di III-V cohorts.

Indeed, patients developing Clavien-Dindo grade III-V complications had significantly lower PD compared to those with no or Clavien-Dindo I-II complications after both colon and rectal resections. However, TAMA and TPA failed to show a statistical difference, which is contrary to the conclusions of some of the previously reported studies that suggested that lean muscle mass is a predictor of major surgical complications [9]. Statistical error due to limited sample size may be an explanation for this observation. At the same time, it can be speculated that TAMA and TPA only reflect muscle mass, while PD rather reflects muscle quality, thus adhering better to the updated sarcopaenia definition [4]. In other words, muscle function may be impaired by fat infiltration that does not necessarily affect its volume. Also of note, from a practical point of view, PD measurement was experienced as simple and quick procedure, calculated automatically in $\mathrm{HU}$ from the $\mathrm{CT}$ scan, while TAMA and TPA measurements were more time consuming, requiring also calculation of the proportion to the square of the patient's height as an additional step. Based on the above, PD might be considered as a favoured sarcopaenic indicator [10].

Obviously, the presented results should be considered as preliminary and interpreted with caution. Due to the unicentric nature of the study, its external validity is limited. Future research is needed to determine cut-off values for PD as a radiological indicator of sarcopaenia. Although different methods have been reported to determine reference standards, including lower quartile of mean density [18] and receiver operating characteristic curve analysis [10], the currently reported thresholds are only arbitrary $[4,16]$. Furthermore, the true value of the PD as a predictor of postoperative complications should be investigated to establish a clear definition of the perceived correlation. Also of note, PD could become a part of more complex predictive tools. This would allow for targeted pre-operative interventions to improve postoperative outcomes (preoperative counselling, nutritional support, prehabilitation) as well as adjustments of surgical strategies in risk-stratified sarcopaenic patients [16]. Laparoscopic colorectal cancer surgery combined with enhanced recovery after surgery protocol seems to reduce the negative impact of sarcopaenia on short-term outcomes and, as such, may be considered relevant [19].

\section{Conclusions}

Psoas density is significantly decreased in patients with Clavien-Dindo grade III-V complications after curative resection for colon and rectal cancer. Due to the simplicity and affordability of its assessment from preoperative staging CT scan, it might be considered as an optimal sarcopenic indicator to be utilised in everyday practice. Future studies are needed to determine PD cut-off values indicating sarcopaenia, thus allowing for its accurate validation as a prognostic factor for postoperative complications.

\section{Acknowledgments}

This study was supported by Specific University Research Grant no. MUNI/A/1307/2019 provided by the Ministry of Education, Youth, and Sports of the Czech Republic.

\section{Conflict of interest}

The authors declare no conflict of interest.

\section{References}

1. Rawla P, Sunkara T, Barsouk A. Epidemiology of colorectal cancer: incidence, mortality, survival, and risk factors. Gastroenterology Rev 2019; 14: 89-103.

2. Pak H, Maghsoudi LH, Soltanian A, et al. Surgical complications in colorectal cancer patients. Ann Med Surg 2020; 55: 13-8.

3. Dindo D, Demartines N, Clavien PA. Classification of surgical complications. A new proposal with evaluation in a cohort of 6336 patients and results of a survey. Ann Surg 2004; 240: 205-13.

4. Cruz-Jentoft AJ, Bahat G, Bauer J, et al. GUIDELINES sarcopenia: revised European consensus on definition and diagnosis. Age Ageing 2019; 48: 16-31.

5. Goodpaster BH, Kelley DE, Thaete FL, et al. Skeletal muscle attenuation determined by computed tomography is associated with skeletal muscle lipid content. J Appl Physiol 2000; 89: 104-10. 
6. Aubrey J, Esfandiari N, Baracos VE, et al. Measurement of skeletal muscle radiation attenuation and basis of its biological variation. Acta Physiol 2014; 210: 489-97.

7. Martin L, Birdsell L, Macdonald N, et al. Cancer cachexia in the age of obesity: skeletal muscle depletion is a powerful prognostic factor, independent of body mass index. J Clin Oncol 2013; 31: 1539-47.

8. Berkel AEM, Klaase JM, de Graaff F, et al. Patient's skeletal muscle radiation attenuation and sarcopenic obesity are associated with postoperative morbidity after neoadjuvant chemoradiation and resection for rectal cancer. Dig Surg 2019; 36: 376-83.

9. Jones KI, Doleman B, Scott S, et al. Simple psoas cross-sectional area measurement is a quick and easy method to assess sarcopenia and predicts major surgical complications. Colorectal Dis 2015; 17: 020-6.

10. Herrod PJJ, Boyd-Carson H, Doleman B, et al. Quick and simple; psoas density measurement is an independent predictor of anastomotic leak and other complications after colorectal resection. Tech Coloproctology 2019; 23: 129-34.

11. Zhang WT, Lin J, Chen WS, et al. Sarcopenic obesity is associated with severe postoperative complications in gastric cancer patients undergoing gastrectomy: a prospective study. J Gastrointest Surg 2018; 22: 1861-9.

12. Gaillard M, Tranchart H, Maitre S, et al. Preoperative detection of sarcopenic obesity helps to predict the occurrence of gastric leak after sleeve gastrectomy. Obes Surg 2018; 28: 2379-85.

13. Sayer A, Syddall H, Martin H, et al. The developmental origins of sarcopenia. J Nutr Health Aging 2008; 12: 427-32.

14. Beaudart C, McCloskey E, Bruyere O, et al. Sarcopenia in daily practice: assessment and management. BMC Geriatr 2016; 16: 170.

15. Mourtzakis M, Prado CMM, Lieffers JR, et al. A practical and precise approach to quantification of body composition in cancer patients using computed tomography images acquired during routine care. Appl Physiol Nutr Metab 2008; 33: 997-1006.

16. Jones K, Gordon-Weeks A, Coleman C, et al. Radiologically determined sarcopenia predicts morbidity and mortality following abdominal surgery: a systematic review and meta-analysis. World J Surg 2017; 41: 2266-79.

17. Engelge K, Museyko O, Wang L, et al. Quantitative analysis of skeletal muscle by computed tomography imaging - state of the art. J Orthop Translat 2018; 15: 91-103.

18. Trotter J, Johnston J, Ng A, et al. Is sarcopenia a useful predictor of outcome in patients after emergency laparotomy? A study using the NELA database. Ann R Coll Surg Engl 2018; 100: 377-81.

19. Pedziwiatr M, Pisarska M, Major P, et al. Laparoscopic colorectal cancer surgery combined with enhanced recovery after surgery protocol (ERAS) reduces the negative impact of sarcopenia on short-term outcomes. Eur J Surg Oncol 2016; 42: 779-87.

Received: 25.09.2020, accepted: 16.10.2020 